insbesondere für kleine und mittlere Unternehmen zumindest kurz- und mittelfristig prohibitiv wirken, z.B. wenn umweltverträgliche Inputs eingesetzt werden müssen, die im Lande nicht hergestellt werden (etwa Farben und Lacke). Drittens ist die Vergabe von Umweltzeichen an Prüfverfahren in den Vergabeländern gebunden; die fehlende Anerkennung von Laborergebnissen aus Entwicklungsländern bzw. das Fehlen solcher Labors erschweren und verteuern den Zugang zu den Umweltzeichen erheblich.

Als Lösungsansatz wird die Einführung von eigenen Programmen zur Umweltkennzeichnung in Entwicklungsländern propagiert. Dies hat aber nur Sinn, wenn die Nachfrage nach gekennzeichneten Produkten auf dem Binnenmarkt ein bestimmtes Volumen erreicht hat, oder wenn Umweltzeichen für Exportprodukte in den Abnehmerländern auch anerkannt werden. Letzteres ist sehr schwierig, weil in den zuständigen Stellen der OECD-Länder detailliertes (Erfahrungs-)Wissen über Produktions- und Umweltbedingungen in Entwicklungsländern meist fehlt.

Insgesamt kann die Durchsetzung nachhaltiger Produktions- und Konsummuster im Norden die Einführung umweltverträglicheren Wirtschaftens auch im Süden fördern; dies ist allerdings an die Voraussetzung gebunden, dafür keine protektionistischen Instrumente, sondern positive Anreize einzusetzen sowie finanzielle und personelle Mittel zur Förderung notwendiger politischer und technologisch-organisatorischer Lernprozesse in Entwicklungsländern bereitzustellen.

\section{Anmerkungen}

(1) Vgl. Jürgen Wiemann et al.: Ecological Product Standards and Requirements as a New Challenge for Developing Countries' Industries and Exports. The Case of India's Leather, Textile and Refrigeration Industries. DIE, Berlin 1994 und Imme Scholz: Umweltverträglicher Außenhandel: Empirische Erfahrungen aus drei chilenischen Exportbranchen, in: Nord-Süd-Aktuell. Bd. 9, H. 1, 1995.

(2) Dies betrifft Hersteller aus Kanada, Finnland und Brasilien. Vgl. UNCTAD: Newly Emerging Environmental Policies with a Possible Trade Impact: A Preliminary Discussion. Genf 1995, S. $12 f$

\section{Die Autorin}

Imme Scholz ist Diplom-Soziologin und urbeitet als wissenschaffliche Mitarbeiterin beim Deutschen Institut für Entwicklungspolitik, Hallerstr. 3 10587 Berlin, Tel: 030-390730

Umrisse einer Strategie zur Förderung „nachhaltigen Konsums”

\title{
Agenda 21 und Studien über nachhaltige Entwicklung
}

\section{Auf der Basis der heute bereits vorhandenen Erfahrungen des Umweltbundes- amtes im Fachgebiet "Methoden der Produktbewertung, Umweltzeichen" las- sen sich einige Punkte zur Fortentwicklung des nachhaltigen Konsumverhaltens zusammenstellen. Dabei wird darauf verzichtet, die bereits eingeleiteten und derzeit geplanten Initiativen des Umweltbundesamtes darzustellen.}

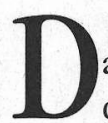
Won Harald Neitzel as Thema „Umwelt und Konsum“ hat vor dem Hintergrund der Aufnahme eines Kapitels 4 ,Veränderung der Konsumgewohnheiten" in der Agenda 21 der UNCED 1992 in Rio neuen Auftrieb erhalten. Insbesondere besteht jetzt die Chance, die Bandbreite dieses Themas und seine Bedeutung für eine nachhaltige Entwicklung systematisch aufzubereiten und die mittel- und langfristig, aber auch die kurzfristig notwendigen Maßnahmen für ein ökologischen Zielen verpflichtetes Konsumverhalten mit den beteiligten Kreisen intensiv zu diskutieren.

Die für eine breite Diskussion eines nachhaltigen Konsums notwendigen wissenschaftlichen Untersuchungen stehen bereits zur Verfügung. Sie thematisieren zum Beispiel die Erforderlichkeit von Umweltzielen $(1,4)$, die Notwendigkeit einer ethischen Fundierung (2), die Verständigung auf neue Leitbilder und eine Veränderung der Bedürfnispräferenzen (3,4). Diesé Arbeiten erbringen allesamt wichtige Beiträge für eine langfristig orientierte Strategiediskussion. Ihre Mängel bestehen jedoch vor allem darin, sich mit bereits vorhandenen Instrumenten/Initiativen $\mathrm{zu}$ wenig auseinanderzusetzen, diese zum Beispiel zu evaluieren und auch kurzfristig orientierte Verbesserungsvorschläge $z u$ unterbreiten (5). Sie entlasten daher alle beteiligten Kreise nicht von der Aufgabe, sich auf nächste Schritte und Prioritäten im Rahmen der zur Verfügung stehenden finanziellen und organisatorischen Rahmenbedingungen $\mathrm{zu}$ verständigen. Auch setzen sie sich nicht mit den zunehmend restriktiver werdenden Rahmenbedingungen einer nachhaltigen Entwicklung auseinander (Arbeitslosigkeit, soziale Sicherheit, Altersentwicklung, Kriminalität, Immigration) und mit den sich hieraus ergebenen Fragen der zukünftigen Prioritätensetzungen, sowohl im politischen
Raum als auch in der Präferenz privater Haushalte für Aufmerksamkeiten, Bedürfnisse und Budgetverwendung.

\section{Konvention beteiligter Kreise}

Angestrebt werden sollte eine Konvention der beteiligten Kreise (insbesondere Konsumgüterindustrie, Handel, Verbraucher- und Umweltverbände, Regierung und Verwaltung) über die Notwendigkeit zur Entwicklung und Förderung nachhaltigen Konsumverhaltens. Diese Konvention könnte zum Beispiel folgende Punkte beinhalten (6):

- Bedeutung der Kategorien Umweltbewußtsein, Umweltverhalten, umweltbewußtes Management

- nachhaltigen Konsum als einen essentiellen Bestandteil des Leitbildes einer nachhaltigen Entwicklung darzustellen

- die Förderung des „nachhaltigen Konsums“ als "Gemeinschaftsaufgabe“ von Staat und Gesellschaft darzustellen; die besonderen Verantwortlichkeiten von Industrie und Handel, Regierung und Verwaltung, Kommunen, Umweltverbände, Verbraucherverbände, Gewerkschaften und Kirchen kurz zu umreißen

- die Notwendigkeit der Entwicklung von Zielen und Zwischenzielen (Leitindikatoren umweltgerechten Konsumverhaltens) ist zu betonen;

- die besondere Bedeutung von Handlungsangeboten und der hierfür erforderlichen Bereitstellung der Infrastruktur, von Infomation und Beratung herauszustellen.

\section{- Wissenschaffliche Fundierung}

Notwendig ist ferner eine bessere wissenschaftliche Fundierung der Prioritäten, Effekte und Potentiale umweltgerechten Verbraucherverhaltens. Wichtige Bausteine hierfür sind: 
- Ökobilanzen, zum Beispiel wäre die spezifische Bedeutung der Herstellungs-, Nutzungsund Entsorgungsphase von Produkten herauszuarbeiten

- Berechnungen des Umweltentlastungspotentials von Handlungsempfehlungen, die zugleich eine Basis von Zielen, Zwischenzielen und Erfolgskontrollen sein können, z.B. Verzicht auf Stand-By-Betrieb, ökologisch richtige Waschmitteldosierung oder

- hierdurch zugleich Aufwertung von „Marginalien“ (Vergleich mit anlagenbezogenen Entlastungspotentialen)

- Verständigung über einen Kanon an Leitindikatoren für die Entwicklung des Konsumverhaltens im Hinblick auf Nachhaltigkeitsziele.

\section{- Neve Themen erschließen}

Zugleich sind neuere Themen als Bausteine nachhaltigen Konsumverhaltens zu erschließen und zu aktivieren, zum Beispiel beim Handel oder in der umweltbezogenen Verbraucherberatung. Von besonderer Bedeutung sind hierbei die Ergebnisse von Modellprojekten und -erfahrungen:

- Förderung einzelner Bausteine nachhaltigen Konsumverhaltens: Verleihangebote, SecondHand-Markt, Nutzung von Dienstleistungen statt Kauf von Produkten, Sharing, Leasing

- regionale Produkte (Herkunftskennzeichnungen, Kampagnen)

- Herausstellung langlebiger Produkte (Garantieleistungen, Reparaturleistungen)

- Fortentwicklung von Rücknahmestrukturen, recyclinggerechter Konstruktion und Verwertung

- Einbeziehung ökologisch und sozial akzeptable Produkte aus Entwicklungsländern.

\section{Produktkennzeichnung}

Aufgrund der bisher erbrachten wichtigen Beiträge zu einer ökologischen Verbesserung der Produkte sind die bewährten und begonnenen Initiativen und Instrumente der Produktkennzeichnung fortzuentwickeln. So zum Beispiel:

\section{Der Autor}

Harald Neitzel leitet das Fachgebiet "Methoden der Produkibewertung, Umweltzeichen ${ }^{n}$ im Umweltbundesamt

Kontakt: Umweltbundesamt, Postfach 3300 22,

14191 Berlin, Tel. 030/23145-703, Fax. 23145-787
- Umwelttests von Produkten und Dienstleistungen (Stiftung Warentest, Öko-Test)

- Umweltzeichen „Blauer Engel“, Europäisches Umweltzeichen und andere umweltbezogene Kennzeichen, wie z.B. im Rahmen der AGÖL, für Produkte aus Entwicklungsländern oder im Textilbereich

- Fortentwicklung der Deklarationsregelungen und Informationspflichten einschl. Verhaltensgebote für Produkte

- Ausbau freiwilliger Informationssysteme wie z.B. Produktpaß

- Empfehlungen zur Gestaltung von Umweltargumenten in der Werbung.

\section{- Weniger Ordnungsrecht}

Alle Diskussionen über die Förderung nachhaltigen Konsumverhaltens sollten von einem Bedeutungsrückgang klassischer ordnungsrechtlicher Instrumente (Ge- und Verbote, Genehmigungsverfahren) ausgehen. Ausschlaggebend hierfür ist eine Reihe von Gründen, die auch mittelfristig bestehen bleiben dürften, wie zum Beispiel die Orientierung am Europäischen Binnenmarkt und dem hieraus erwachsenen Zwang, europäische Regelungen anzustreben, die zunehmende Bedeutung freiwilliger Regelungen und staatsentlastender Maßnahmen in der Umweltpolitik oder die grundsätzliche Einsicht, daß ökologisch gewünschte Verhaltensweisen nur dann erfolgreich sein können, wenn sie gewollt und nicht staatlich verordnet werden.

\section{- Finanzielle, nicht-materielle Anreize}

Eine große Bedeutung auch vor dem Hintergrund der Diskussion um die Einführungen von ÖkoSteuern - kommt der Fortentwicklung finanzieller und nicht-materieller Anreize zu. Allerdings sind auch hier einschlägige Maßnahmen, z.B. zur Besteuerung des Energieverbrauchs, vom politischen Willen abhängig.
Vor finanziellen Anreizen als Allheilmittel ist allerdings zu warnen, zumal die derzeit bestehenden einschlägigen Handlungsmöglichkeiten, Heizöl, Strom oder auch Benzin einzusparen, noch lange nicht ausgeschöpft werden.

\section{Ökologische Bewußtseinsbildung}

Weitgehende Ratlosigkeit herrscht noch, wie einige der Voraussetzungen zur Förderung nachhaltigen Konsumverhaltens, die „ökologische Bewußtseinsbildung, Umwelterziehung und Verbrauchermotivierung“, über die bestehenden Initiativen hinaus gefördert werden können. Von besonderer Bedeutung ist hierbei die Auswertung der einschlägigen sozialwissenschaftlichen Forschung, auch zum Zusammenhang von Umweltbewußtsein und Umweltverhalten (7). In diesem Zusammenhang ist insbesondere auch eine Auseinandersetzung mit den hemmenden Faktoren nachhaltigen Verbraucherverhaltens notwendig:

- Informationsbarrieren

- Verhaltensbarrieren

- Wahrnehmungsbarrieren

- Gefiihlsbarrieren (psychische Abwehrmechanismen)

\section{... für denkende Anleger(innen) ...}
Sie wollen Ihr Geld rentabel und ökologisch sinnvoll anlegen?

Der Informationsdienst ÖKO-INVEST liefert Ihnen dazu alle zwei Wochen aktuelle und umfassende Informationen, z.B. über alternative Banken, aussichtsreiche ,grüne“ Aktien oder ökologische Beteiligungsmöglichkeiten in Investmentfonds bzw. nicht börsennotierten Gesellschaften. Damit Sie wissen, welche Unternehmen sauber sind und es auch bleiben.

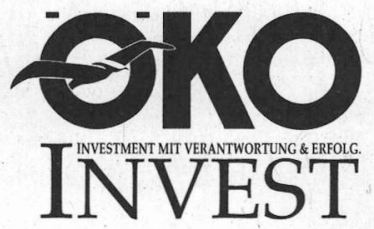

Ihr kostenloses Probeexemplar oder Infos zum Jahrbuch „Grünes Geld" können Sie per Postkarte oder Fax abrufen bei der

\section{ÖKO-INVEST Verlags-GmbH \\ Schweizertalstr. 8-10/5 \\ A-1130 Wien}

Tel. 0043/1/535 1815

Fax 0043/1/535 4669 
- gesellschaftliche Barrieren (,außengeleitete Orientierungen").

\section{Neve Wohlstandsbilder}

Dieser Überlegung folgend muß derzeit die Wahrscheinlichkeit des Eintreffens bzw. der Akzeptanz von neuen Wohlstandsbildern und -orientierungen als „offene Flanke“ einer Strategie für einen nachhaltigen Konsum bewertet werden (8). Hilfreich für die weitere Diskussion erscheint in diesem Zusammenhang eine Verständigung über die Leitbilder ,nachhaltigen Konsumverhaltens" in den verschiedenen Handlungsbereichen des Verbraucherverhaltens. Dabei bietet es sich - ausgehend von der derzeitigen praktischen Situation - an, von einer „mainstream"- und einer ,pioneer"-Orientierung zu sprechen. Unter „mainstream“ würde man dabei all die Verhaltensweisen zusammenfassen, die von einer Mehrheit der Bevölkerung erwartet werden können, z.B. die Bildung von Fahrgemeinschaften im Berufsverkehr. Unter „pioneers" würden all die Initiativen laufen, die derzeit lediglich von wenigen besonders bewußten Verbrauchern erwartet werden können, z.B. die Beteiligung am Car-Sharing. Wichtig ist jedoch die „Vision“, daß das Pionierverhalten von heute in den „mainstream“ von morgen wachsen kann und muß (9).

\section{Anmerkungen}

1) Friends of the Earth Netherlands: Action Plan Sustainable Netherlands, Amsterdam 1.993

2) Der Rat von Sachverständigen für Umwelffragen: Umweltgutachten 1994 und 1996

3) Enquete-Kommission "Schutz des Menschen und der Umwelt" des Deutschen Bundestages: Die Industriegesellschaft gestalten - Perspektiven für einen nachhaltigen Umgang mit Stoff- und Materialströmen, 1994

4) Wuppertal Institut für Klima, Umwelt, Energie GmbH: Zukunftsfähiges Deutschland, 1995

5) Ein Überblick über die Bandbreite der Themen des nachhaltigen Konsums vermittelt z.B. der Text: Das Umweltverhalten der Verbraucher, Daten und Tendenzen, UBA-TEXTE 75/94 (kostenlos anzufordern).

6) Die Diskussion über eine entsprechende Konvention der beteiligten Kreise wurde auf einem Fachgespräch im Umweltbundesamt am 9./10. Mai 1996 begonnen und soll fortgesetzt werden.

7) Das Umweltbundesamt hat zur Vorbereitung eines "Demonstrationsvorhabens" nachhaltiger Konsum beim Institut für sozialökologische Forschung, Frankfurt a.M. die Studie "Ergebnisse der sozialwissenschaftlichen Umwelfforschung zu den Themenbereichen Umweltbewußtsein, Umweltverhalten, Lebensstile und Wertewandel" in Auftrag gegeben; diese soll noch 1996 veröffentlicht werden.

8) Das Umweltbundesamt geht in seinem derzeit noch in Arbeit befindlichen Bericht: Schritte zu einem "nachhaltigen Deutschland" in ausgewählten Bereichen (Mobilität, Nahrungsmittel, Textilien) von einem "Effizienzszenario" und einem "Struktur- und BewuBtseins-Szenario" als Voraussetzungen für eine nachhaltige Entwicklung aus.

9) Das Umweltbundesamt hat hierzu - gleichfalls zur Vor-

\section{Literaturliste zum Themenbereich „Nachhaltiger Konsum"}

Beier, U.: Der fehlgeleitete Konsum. Eine ökologi sche Kritik am Verbraucherverhalten. Frankfurt am Main 1993

Commission On Sustainable Development Changing consumption and production patterns. Report of the Secretary-General. United Nations, Economic and Social Council 1996

Dammann, R., Strickstrock, F.: Der Unternehmenstester. Die Lebensmittelbranche. Ein Ratgeber für den verantwortichen Einkauf. Imug Institut für Markt-UmweltGesellschaft. Hamburg 1995

Einert D., Schrader U.: Die Bedeułung des Eigenfums für eine Ökologisierung des Konsums. Lehr- und Forschungsbericht Nr. 36 des Lehrstuhls Markt und Konsum, Hrsg. v. Prof. Dr. U. Hansen, Hannover 1996, 160 Seiten, 25 Mark

Forschungsgruppe Konsum und Verhalten: Konsumentenforschung. Gewidmet Werner Kroeber-Riel zum 60. Geburtstog. München 1994

Hellenbrandt, S./Rubik, F.: Produkt und Umwelt. Anforderungen, Instrumente und Ziele einer ökologischen Produktpolitik. Marburg 1994

Raabe T.: Retro marketing. Begründung und Program matik eines Forschungsprojekts zum Beitrag des Marketing bei der Realisation von "Kreislaufwirtschaften" als umweltpolitisches Leitkonzept, Lehr und Forschungsbericht Nr. 32 des Lehrstuhls Markı und Konsum, Hisg. v. Prof. Dr. U. Hansen, Hannover 1995, 50 Seiten, 10 Mark

Reddift, M.: Wasted. Counting the Cost of Global Consumption. Earthscan, London 1995

Spiller, A.: Ökologieorientierte Produktpolitik. Forschung, Medienberichte und Marktsignale. Marburg 1996

Schrader Ulf: Der ökologisch bedingte Produktnutzen. Lehr- und Forschungsbericht Nr. 30 des Lehr- bereitung des unter (7) angekündigten Demonstrationsvorhabens - beim Sekretariat für Zukunftsforschung / Clearing House for Applied Future eine Zusammenstellung und Auswertung von Initiativen mit einem Bezug zur Entwicklung nachhaltigen Konsumverhaltens in Auftrag gegeben. Diese soll gleichfalls noch 1996 veröffentlicht werden. stuhls Markt und Konsum, Hisg. v. Prof. Dr. U. Hansen, Hannover 1995, 30 Seiten, 10 Mark

Statistisches Bundesamt: UGR-Materialien. Beitrüge zur Umweltökonomischen Gesamtrechnung. Heft 3: Umweltschutzmaßnahmen der privaten Haushalte. Wiesbaden 1994

Steffen, D.: Welche Dinge braucht der Mensch? Hintergründe, Folgen und Perspektiven der heutigen Alltagskultur. Deutscher Werkbund Hessen, Giessen 1995

Stiftung Verbroucherinstitur: Nachhaltiger Konsum aber wie? Dokumentation eines Workshops der Stiffung Verbraucherinstitut. Berlin 1995

SustainAbility Ltd.: Who needs it? Market Implications of Sustainable lifestyles. A Sustain Ability Business Guide. London 1995

Umweltbundesamt: Das Umweltverhalten der Verbraucher. Daten und Tendenzen. Empirische Grundlagen zur Konzipierung von ${ }_{n}$ Sustainoble Consumption Patterns". Elemente einer „Ökobilanz Haushalte“. Berlin, 1994

Verbraucher Initiative $(\mathrm{Hg}$.$) : Konsumwende - mehr$ Wohlstand für alle? Hercusforderungen für eine ökologische und soziale Verbraucherpolitik. Bezug: VI Verlagsund Handel GmbH, Breite Straße 51, 53111 Bonn

Voß, C.: Kann denn Mode "öko" sein? Einkaufsleitfaden Naturtextilien. Wissenschaftsladen Bonn e.V. (Hisg.), Bonn 1995

Weskamp, C. (Hg.) : Ökologischer Konsum. Ansätze und Leitbilder nachhaltig ökologischer und sozialvertröglicher Lebensweisen. Berlin 1995

Hinweis:

Es ist geplant, die Beiträge dieses Schwerpunktes ungekürzt und erweitert um einige Beitrüge, die in diesem Heft nicht mehr veröffentlicht werden konnten, als IÖW-Diskussions-papier herauszubringen. 
(c) 20I0 Authors; licensee IÖW and oekom verlag. This is an article distributed under the terms of the Creative Commons Attribution Non-Commercial No Derivates License (http://creativecommons.org/licenses/by-nc-nd/3.o/), which permits unrestricted use, distribution, and reproduction in any medium, provided the original work is properly cited. 